\title{
Towards a Fire Alarm Model Based on Variable Learning Rate Algorithm with Weight Factor
}

\author{
Wei Wang \\ College of Computer Science and Technology \\ Changchun University \\ Changchun, China \\ e-mail: wangweitea@163.com \\ Lei $\mathrm{Xu}$ \\ College of Optical and Electronical Information \\ Changchun University of Science and Technology \\ Changchun, China \\ e-mail:285138396@qq.com
}

\author{
Li-ping Yang \\ College of Computer Science and Technology \\ Changchun University \\ Changchun, China \\ e-mail: yanglipingtea@163.com
}

\begin{abstract}
Fire alarm with Neural Network (NN) can learn knowledge from multiple sensor data fusion. By adjusting network weight, more stable fire alarm can be achieved. Classic BP NN is likely to fall into local minimum. To address this problem, a Variable Learning Rate Algorithm with Weight Factor (VLRA-BP) was proposed and introduced into automatic intelligent decision in fire alarm. The model with VLRA-BP algorithm uses temperature and smoke sensors to perform intelligent information transformation, so as to achieve target of timely alarming and decrease system false alarm rate. Simulation experiment result shows that system accuracy and average error can effectively monitor simulated fire scene and forecast fire.
\end{abstract}

Keywords- fire alarm; neural network; variable learning rate; weight factor

\section{INTRODUCTION}

Currently, majority of fire alarm systems use combination of various sensors to gather many effective fire parameters and use modern data fusion technology for forecasting fire. Modern data fusion algorithms, such as artificial intelligence, fuzzy logic, genetic algorithms, etc., make up for shortcomings of the classical algorithms, effectively improving accuracy of the fire alarm system and guiding fire alarm system develop toward intelligent direction [1-4]. However, as current intelligent fire alarm systems as concerned, there are still problems of poor system re-liability and stability, single detection signal processing, low effective rate and low warning intelligence. In practical applications, there is still conventional fire alarm system merely using single sensor, which cannot achieve full area coverage detection, resulting in false, leak alarm and so on. It cannot meet the system stability and reliability requirements. Meanwhile, vast majority of fire alarm systems aim at combustion stage alarm, but cannot fire early warning in the smoldering stage. Furthermore, the intelligence level of a fire alarm system is limited, majority of which cannot effectively automatic decision.
The neural networks can achieve multi-sensor data fusion and sensor error compensation. Conclusive results that reflect the characteristics of the data can only be obtained through large amounts of data and processing operations $[5,6]$. In practical applications of multi-sensor data fusion, neural network learning and rely on its strong organizational skills. According to the specific needs of the actual situation, the knowledge of multiple sensor fusion is learned to constantly adjust network weight and threshold, strength stability of data fusion, so as to achieve actual needs. The application of neural network into data fusion needs to establish corresponding network model in accordance with design requirements. Then, the specific learning algorithm should be selected to train the established network model. The matured network will automatically generate automatically generate network weight and threshold, so as to apply matured neural network model into actual fire alarm. In this paper, the classic BP neural network is easy to fall into local minimum problem to form the Variable Learning Rate Algorithm with Weight Factor (VLRA-BP). This model uses temperature, smoke and other sensors data for intelligent information conversion, so as to achieve a timely alarm. The system reduces the false alarm rate for the specific purpose. The paper is arranged as follows: section 2 gives an improved VLRA-BP algorithm; section 3 devises fire warning model according to VLRA-BP algorithm and section 4 concludes our work.

\section{IMPROVED VLRA-BP ALGORITHM}

\section{A. Algorithm Analysis}

BP network can approximate any nonlinear function with any accuracy. Such remarkable advantage makes BP network has been more and more widely used. However, the standard BP algorithm in the application also exposes many shortcomings, specifically in the following areas:

$a$. Completely unable training. This is mainly reflected in the emergence of network paralysis. When it is the right value adjustment is too large during network training, it 
may make all or most of a weighted sum of neurons too large, which makes the input of activation function work in the saturated zone of S-type activation function, leading to very small derivative value. In the calculation of weighted correction amount, since the $\delta$ is proportional to $f^{\prime}$, so when $f^{\prime} \rightarrow 0$, there is $\delta \rightarrow 0$, making the $w \rightarrow 0$. It is equivalent to weight adjustment process almost a halt. In order to avoid the occurrence of this phenomenon, there are usually commonly two ways used. Firstly, select a smaller initial weight. The second is a smaller learning rate, but which increases the training time.

$b$. Too many training times. It has low learning efficiency and slow convergence, which requires a longer training time. For some complex issues, BP algorithm may have to be more than a dozen hours or longer training, which is mainly due to too low local minimum learning rate.

c. Easy to form a local minimum but not the global optimum. BP algorithm allows the network weights converge to a solution, but it does not guarantee that seeks solutions to the global minimum error hyper-plane, which is likely to be a local minimum. This is because the BP algorithm uses a gradient descent and training starts from the slope of the error function along gradually reach the minimum error [7, 8]. For complex networks, the error function is curved multidimensional space, like a bowl, the bowl is its minimum point. However, this bowl is uneven surface, and thus in the course of their training, it may fall into a small valley area, and this produces a small valley area is a local minimum. Thus changing point in all directions so that errors are increased, resulting in training cannot escape the local minima. To solve the above drawbacks, the paper uses a VLRA-BP algorithm to train the BP neural network.

\section{B. Variable Learning Rate Algorithm}

In the standard BP algorithm, the learning rate is generally fixed. However, due to the complexity of the error surface, the error surface for connection weights vary in different regions will exhibit different characteristics, specific learning rate cannot all fit on the error surface area, resulting in BP algorithm converges slowly.

To improve performance and accelerate convergence process, while not increase the complexity and training time, we use a variable learning rate algorithm. In the variable learning rate algorithm, learning rate is adaptively adjusted in iterative process, so that training the neural network is automatically set different rates at different stages of learning. The formula is the learning rate adjustment formula:

$$
\eta(k+1)= \begin{cases}1.05 \eta(k) & E(k+1)<E(k) \\ 0.7 \eta(k) & E(k+1)>1.04 E(k) \\ \eta(k) & \text { others }\end{cases}
$$

From (1) we can see that when the new error exceeds a certain multiple of the old, learning rate will decrease; when a new error is larger than the old error, the learning rate would be increased. Otherwise the learning rate is constant. This approach ensures that the network always maximum acceptable training learning rate.

\section{BP Learning Algorithm with Weighted Factor}

Since the standard BP algorithm adjust the weights only at the current time error gradient descent direction of adjustment, without considering the gradient direction of the previous time, it often makes the training process oscillation and slow convergence [9]. In the paper, in order to improve the training speed of the network, the BP learning algorithm with weighted item is used.

The BP learning algorithm with weighted factor just adds an item that is proportion to former weight variable to each weighted adjustment. Therefore, this paper sets a variable. After each adjustment completed, the adjustment amount is assigned to variable to be used in weighting adjustments below. The adjustment equation with the weighted equation is

$$
\Delta w(k+1)=-\eta \frac{\partial E}{\partial w}+\alpha \Delta w(k)
$$

Where, $\alpha$ is weighted factor. It can be seen from the above equation that the increased weighted factor items that adjust the amount of weight that was once part of superposition to remove this weight adjustment amount. The factor term adjustment reflects the previously accumulated experience of the present moment to adjust the damping can play a role, will not have a big swing.

The essence of weighted item means the value of $\eta$ in learning process is no longer a constant value, but changing. When the train enters the error function hyperplane flat areas, the error will be little change, then

$$
\Delta w(k+1) \approx \Delta w(k)=-\frac{\eta}{1-\alpha} \cdot \frac{\partial E}{\partial w}
$$

The coefficient $\frac{\eta}{1-\alpha}$ in (3) will enable weight adjustment escape from saturated zone and accelerate learning rate.

\section{FIRE ALARM MODEL BASED ON VLRA-BP}

Based on VLRA-BP algorithm, the paper proposes a data fusion system mode consisted by sensor portion, information fusion pretreatment section, information fusion and decision-making data constitute part of the control section and others. On the basis of obtaining certain amount of information, the fusion criteria and algorithms are used to fuse obtained information so as to reach more useful information. The system model includes sensor detecting sub-model, information processing and decision support subsystem, as shown in Fig 1. 


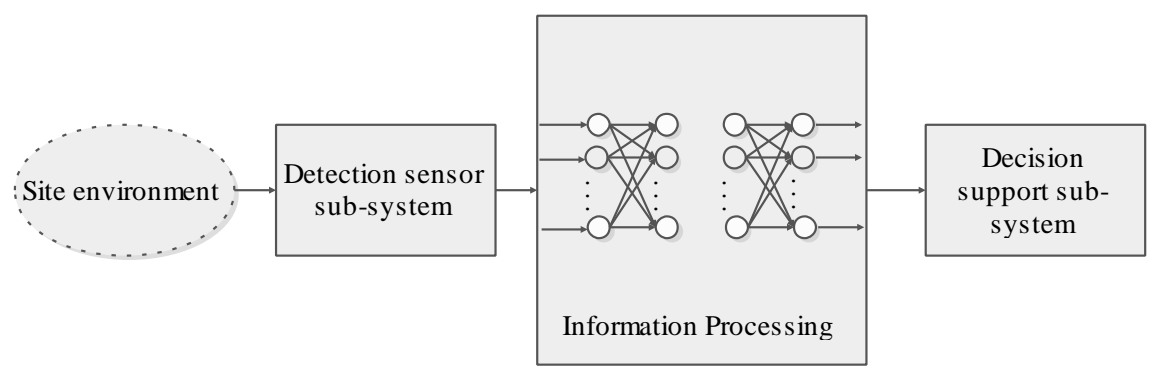

Figure 1. System alarm decision model.

\section{A. Model Construction}

The fire scene real-time dynamic monitoring system uses computers and other advanced technology at different points in time, different classes and different ways of expressing information. In accordance with certain criteria, it applies appropriate method for temperature, smoke, $\mathrm{CO}$ and other sensor data, comprehensive analysis, the final the disaster to make timely and correct judgment [10]. The model uses multi-sensor information to detect, which is integration of hardware-based models, multi-source heterogeneous information obtained is its integration process object, information fusion processing methods and algorithms are the core of information fusion.

In the fire warning decision model including multisensor detection subsystem, pretreatment subsystem integration, information integration and information decision support subsystem, the sensor detection system is the hardware foundation of the entire model, providing information to be processed. Information fusion pretreatment system and information fusion system are the core of model, which is responsible for integrated processing and decision to get the information they need to provide a good input information for decision support systems. Decision support system receives information from the fusion system integration. As a result, experts in vulnerability assessment and decision-making, and ultimately effective decision-making and trigger a series of actions.

\section{B. Model Parameter Settings}

In the fire alarm system in the decision-making model, the core algorithm is VLRA-BP proposed in this paper. With VLRA-BP algorithm, it first needs to determine VLRA-BP model parameters. For VLRA-BP neural network, the input layer for receiving external raw data directly, it is the role of buffer memory. The dimension of the input vector $d$ restricts the input nodes. The system input signals including temperature signal, signal of carbon monoxide and smoke signals, so the input vector is threedimensional, the number of nodes in the input layer is set to 3 .

The determination of hidden layer node number usually uses test method. Input layer and output layer node number restricts node number of hidden layer. The relationship between them is inseparable, so the hidden layer nodes to a number of factors to determine the overall design of the integrated system. Test method used in this paper to select hidden layer nodes can refer to the following formula

$$
n=\sqrt{n_{i}+n_{0}}+a
$$

Where, $n$ is the node number of the hidden layer; $n_{i}$ represents the node number of the input layer; $n_{0}$ represents the node number of the output layer. The $a$ is natural number between 1 and 10 . Usually, the principle to select hidden layer node number is adding 1-2 nodes to number that can meet problem requirements, so as to accelerate the rate of error decline. The hidden layer node number in fire alarm model in this paper is set 8 at last.

The node number of output layer is determined according to the actual situation. This paper forecasting problem, the output information includes output probability of fire, smoldering fire probability and nonprobability output, so the output layer requires three nodes.

\section{Model Validation}

The training sample in Table 1 was used for fire alarm model validation.

TABLE I. FIRE ALARM MODEL TRAINING SAMPLES.

\begin{tabular}{|c|c|c|c|c|c|c|}
\hline Sample & Smoke & CO & Temperature & $\begin{array}{c}\text { Non- } \\
\text { probability }\end{array}$ & $\begin{array}{c}\text { Smoldering } \\
\text { probability }\end{array}$ & $\begin{array}{c}\text { Fire } \\
\text { probability }\end{array}$ \\
\hline 1 & 0.658 & 1 & 0.6 & 0.05 & 0.05 & 0.9 \\
\hline 2 & 0.421 & 0.48 & 0.2 & 0.15 & 0.7 & 0.25 \\
\hline 3 & 0.526 & 0.68 & 0.1 & 0.05 & 0.7 & 0.25 \\
\hline 4 & 0.658 & 0.74 & 0.1 & 0.1 & 0.65 & 0.25 \\
\hline 5 & 0.789 & 0.8 & 0.2 & 0.05 & 0.75 & 0.2 \\
\hline 6 & 0.789 & 0.8 & 0.1 & 0.05 & 0.85 & 0.1 \\
\hline 7 & 0.526 & 0.14 & 0 & 0.85 & 0.1 & 0.05 \\
\hline 8 & 0.421 & 0.2 & 0.1 & 0.9 & 0.05 & 0.05 \\
\hline 9 & 0.474 & 0.8 & 0.6 & 0.1 & 0.2 & 0.7 \\
\hline 10 & 0.5 & 0.66 & 0.1 & 0.05 & 0.7 & 0.25 \\
\hline
\end{tabular}


As the above training samples as concerned, model structure for learning selects VLRA-BP neural network as above mentioned. After training network for 40 times, the network reaches expected target error. The network convergence speed is faster. At this moment, the obtained training error curve is shown in Fig. 2.

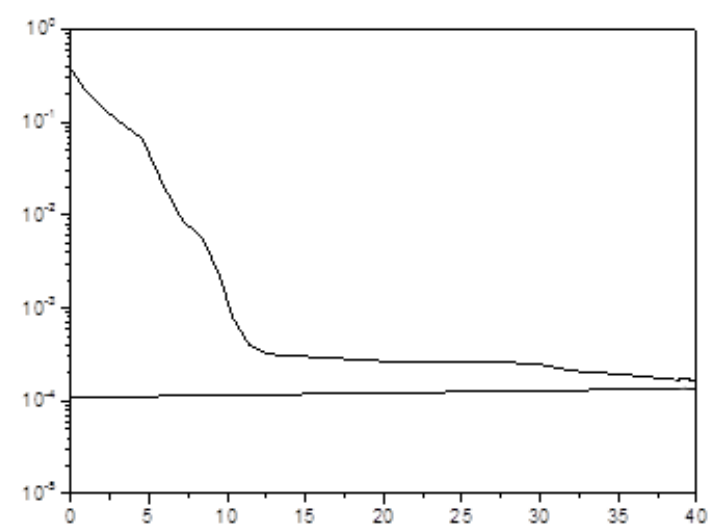

Figure 2. Training error curve.

It can be seen from the figure that this VLRA-BP prediction model can rapidly convergence. With this model, the fire recognition can achieve expected effect, thus improving reliability and credibility of fire alarm prediction detection system. When the hidden neutron number is 8 , the maximum prediction error (root mean squared error) is 0.073 .

\section{CONCLUSION}

Aiming at shortcomings of traditional fire detection system, the paper presents multiple mode data transmission method to combine neural network with data fusion technology, so as to complete fire information analyzing and processing. To address this problem that classic BP neural network is likely to fall into local minimum, a VLRA-BP algorithm was proposed and introduced into automatic intelligent decision in fire alarm. VLRA-BP neural network learning data analysis shows that the system can achieve target of timely alarming and decrease system false alarm rate.

\section{REFERENCES}

[1] Y. Okayama, "A Primitive Study of a Fire Detection Method Controlled by Artificial Neural Net," Fire Safety Journal, vol. 17, no. 6, 1991, pp.535-553.

[2] Wang S., "A trend duration and gradient detector for automatic fire detection," Fire Safety Journal, vol. 27, no. 3, 1996, pp. 45-49.

[3] Naiwei Cheng, Qifeng Wu, “A Decision-Making Method for Fire Detection Data Fusion Based on Bayesian Approach," Proceedings of 2013 Fourth International Conference on Digital Manufacturing and Automation (ICDMA), 2013, pp. 21-23.

[4] Shixin Wang, Yi Zhou, Litao Wang, "A research on fire automatic recognition using MODIS data," Geoscience and Remote Sensing Symposium, vol. 4, no. 5, 2003, pp. 2502-2504.

[5] Susan L., Rose Pehrsson R., Sean J., "Early warning fire detection system us-ing a probabilistic neural network," Fire Technology, vol. 39, 2003, pp. 147-171.

[6] Renee D. Jiji, Mark H., Hammond, "Multi-variable statistical process control for continuous monitoring of networked early warning fire detection (EWFD) systems," Sensors and Actuators, vol. 93, 2003, pp. 107-116.

[7] James A. Milke, Matthew E., "Investigation of multi-sensor algorithms for fire detection," Fire Technology, vol. 39, 2003, pp. 363-382.

[8] Feng Sang, Guo-ping Jiang, Fang Wang, "Compound Intelligent Fire Detector Con-structed By Using BP Network," Process Automation Instrumentation, vol. 3, 2003, pp. 39-41.

[9] Jian-hua Zhao, Jun Fang, Xue-ming Shu, "An Identification Method of Fire Smoke Based on Neural Network," Acta Optica Sinica, vol. 23, no. 9, 2003, pp. 1086-1089.

[10] Hung-Ho Lee, Misra, M., "Early warning of ship fires using Bayesian probability estimation model," Proceedings of the 2005 American Control Conference, 2005, vol. 3, pp. 1637-1641. 